\title{
Existence of a dictatorial subgroup in social choice with independent subgroup utility scales, an alternative proof
}

Anna B. Khmelnitskaya

\begin{abstract}
Social welfare orderings for different scales of individual utility measurement in distinct population subgroups are studied. In Khmelnitskaya and Weymark (2000), employing the continuous version of Arrow's impossibility theorem, it was shown that for combinations of independent subgroups scales every corresponding social welfare ordering depends on the utilities of only one of the subgroups and is determined in accordance with the scale type proper to this dictatorial subgroup. In this article we introduce an alternative completely self-contained proof based on the study of the structure of level surfaces of a social welfare function which provides a real-valued representation of the social welfare ordering.
\end{abstract}

\section{Introduction}

In Arrow's famous impossibility theorem [1], individual preferences are ordinally measurable and interpersonally noncomparable. Building on the seminal work of Sen [14], there is now an extensive literature that investigates the implications for social decision-making of alternative assumptions concerning the measurability and interpersonal comparability of individual preferences. See, for example, Roberts [12], [13], d'Aspremont [3], Yanovskaya [16], [17], Tsui and Weymark [15], Bossert and Weymark [2]. These studies adapt mainly the welfarist approach to social choice and assume that only individual utilities matter for ranking a feasible set of social alternatives. In this case a social choice rule can be equivalently described in terms of a social welfare ordering - a social ordering of the admissible profiles of individual utilities (admissibility is understood as the satisfaction of several a priori appealing conditions), or in terms of a social welfare function - a function that represents a social welfare ordering and measures social welfare. Various assumptions concern-

Anna B. Khmelnitskaya

$\mathrm{SPb}$ Institute for Economics and Mathematics Russian Academy of Sciences, 1 Tchaikovsky St., 191187, St.Petersburg, Russia, e-mail: a.khmelnitskaya@math.utwente.nl 
ing the measurability and interpersonal comparability of utility can be formalized by partitioning the set of feasible individual profiles and requiring the social welfare ordering to be constant over a cell of the partition. These studies show that under different measurability-comparability assumptions over individual utilities, i.e., in case when more democracy is adapted by the society, classes of nondictatorial social choice rules exist that satisfy all of Arrow's axioms (restated in terms of utility functions). In the aforecited publications the measurement scales of individual utilities are assumed to be of the same type across the entire society. An extension of this direction is a study of Arrovian social choice problems when individual utilities in disjoint subgroups of individuals are measured by different scale types, in other words, when separate subgroups of individuals admit different types of information. This situation is common in real decision making. A typical example is the partitioning of a human society into families which in turn consist of individuals. If an outsider is making the comparisons based on reports from individuals, it is reasonable to suppose that the kind of information available within and between families will be different in general. Indeed, the kinds of utility comparisons that can be made within a family cannot be made between people who do not know each other. A number of publications of the author (Khmelnitskaya [7], [9], Khmelnitskaya and Weymark [10]) is devoted to study of Arrovian social choice problems with different scales of individual utility measurement in disjoint subgroups of individuals. In particular, in Khmelnitskaya and Weymark [10] it is shown that for ordinally or cardinally measurable subgroup utility when levels (and in the case of cardinal utilities, differences) of utility may or may not be interpersonally comparable while no utility comparisons between subgroups are possible, every continuous social welfare ordering that meets the weak Pareto principle depends on the utilities of only one of the subgroups and is determined in accordance with the scale type admissible to this dictatorial subgroup. Here we introduce another proof $^{1}$ for this statement restated in equivalent terms of a social welfare function. This proof is longer but completely self-contained different to the proof in [10] which is based on the employment of Bossert-Weymark [2] continuous analogue of both - Arrow's [1] impossibility theorem and Sen's [14] variant of Arrow's theorem for cardinally measurable utilities. Moreover, being based on the study of level surfaces of a social welfare function this proof provides also extra deep insight into the structure of possible interrelations between utilities of different individuals, while the proof in [10] allows only to state existence of a dictatorial subgroup.

In Section 2, we introduce basic definitions and notation and provide a formal statement of the problem. Section 3, provides the proof of the existence of a dictatorial subgroup for different combinations of mutually independent subgroup scales restated in terms of a social welfare function.

\footnotetext{
${ }^{1}$ This proof circulated before in Khmelnitskaya's unpublished manuscript [8].
} 


\section{The framework}

Consider a society consisting of a finite set $N=\{1, \ldots, n\}$ of $n \geq 2$ individuals. Let $X$ be a finite set of at least three alternatives and let $\mathscr{R}$ denote the set of all possible preference orderings over $X$. The members of $\mathscr{R}$ are assumed to be weak orders, i.e., complete, reflexive and transitive binary relations. A social choice problem is a triple $<X, N,\left\{R_{i}\right\}_{i \in N}>$, where $\left\{R_{i}\right\}_{i \in N}$ is a profile of individual preferences $R_{i} \in \mathscr{R}, i \in N$. To introduce measurability/comparability assumptions, we consider individual preferences represented as individual utilities, which may be interpreted as measurements of these preferences. So, let $U$ be the set of all real-valued functions defined on $X \times N$ : for any $u \in U$, let $u(x, i)$ denote the $i$ th individual utility at the alternative $x \in X$. By a solution to a social choice problem we understand a social welfare functional, which is a mapping $f: \mathscr{D} \rightarrow \mathscr{R}$ where $\mathscr{D} \subseteq U$ is the domain of $f$. We assume $f$ satisfies three welfarism axioms:

Unrestricted Domain. $\mathscr{D}=U$, i.e., $f$ is defined for all $u \in U$.

Independence of Irrelevant Alternatives. For any $u, u^{\prime} \in \mathscr{D}$ and $A \subseteq X$, if $u(x, i)=$ $u^{\prime}(x, i)$ for all $x \in A$ and $i \in N$, then $R: A=R^{\prime}: A$ where $R=f(u)$ and $R^{\prime}=f\left(u^{\prime}\right)$. ( $R: A$ denotes the restriction of $R$ to $A \subseteq X$.)

Pareto Indifference. For any pair $x, y \in X$ and for all $u \in \mathscr{D}$, if $u(x, i)=u(y, i)$ for all $i \in N$ then $x I y$, where $I$ denotes the indifference relation corresponding to $R=f(u)$.

According to the welfarism theorem (D'Aspremont and Gevers [4] and Hammond [6]), these three axioms ensure that only individual utilities matter when ranking social alternatives, so any vector $u=\left(u_{1}, \ldots, u_{n}\right)$ in the $n$-dimensional Euclidian space $\mathbb{R}^{n}$ can be considered as a profile of individual utilities for the society $N$; here $u_{i}$ is the utility of $i$ th individual. From this perspective, a solution to a social choice problem can be regarded as a social welfare ordering (SWO), which is a weak order $R^{*}$ on $\mathbb{R}^{n}$, the set of possible profiles of utility vectors. We assume that $R^{*}$ also satisfies the Weak Pareto property.

Weak Pareto (WP). For all $u, v \in \mathbb{R}^{n}$, if $u_{i}>v_{i}$ for all $i \in N$, then $u P^{*} v$, where $P^{*}$ denotes the strict preference relation corresponding to $R^{*}$.

A function $W: \mathbb{R}^{n} \rightarrow \mathbb{R}^{1}$ represents the $\mathrm{SWO} R^{*}$ if for all $u, v \in \mathbb{R}^{n}$

$$
u R^{*} v \Longleftrightarrow W(u) \geq W(v) \text {. }
$$

The representation $W$ is called a social welfare function (SWF). By WP, any SWF $W$ is strictly increasing, i.e., for all $u, v \in \mathbb{R}^{n}$

$$
u \gg v \Longrightarrow W(u)>W(v) .
$$

We impose one more restriction on an SWO $R^{*}$ requiring $R^{*}$ to be continuous.

Continuity (C). For all $u \in \mathbb{R}^{n}$, the sets $\left\{v \in \mathbb{R}^{n} \mid v R^{*} u\right\}$ and $\left\{v \in \mathbb{R}^{n} \mid u R^{*} v\right\}$ are closed in $\mathbb{R}^{n}$. 
Continuity guarantees the existence of a continuous SWF [5].

In the sequel by $D_{n}$, we denote the diagonal of $\mathbb{R}^{n}$. Let for any real $c \in \mathbb{R}^{1}, c_{N}$ be a vector in $\mathbb{R}^{n}$ with all components equal to $c$ and let $g(c)=\left\{u \in \mathbb{R}^{n} \mid W(u)=c\right\}$ be a $c$-level surface of the SWF $W$; obviously, for every $u \in \mathbb{R}^{n}, g(W(u))$ is a level surface of $W$ containing $u$.

Remark 1 Because of continuity and strict monotonicity of all SWF, every level surface of any SWF meets a diagonal $D_{n}$ of $\mathbb{R}^{n}$ and moreover, this meet of set is a singleton. Hence, a natural scale for the meanings of SWF arises: since every SWF $W$ is defined up to monotonic strictly increasing transforms, then without loss of generality it may be assumed that for any $u \in \mathbb{R}^{n}, W(u)=c$, with $c$ defined by the equality $g(W(u)) \cap D_{n}=\left\{c_{N}\right\}$.

In the classic case of Arrow utilities were ordinally measurable and interpersonally non-comparable. More generally, within the SWO framework, the degree of measurability and comparability of utility inside the society $N$ can be specified by a class of invariance transforms $F$, where each transform $f \in F$ is a list of functions $\mathrm{f}=\left\{\mathfrak{f}_{i}\right\}_{i \in N}, \mathrm{f}_{i}: \mathbb{R}^{1} \rightarrow \mathbb{R}^{1}$, with the property: for all $u, v \in \mathbb{R}^{n}$

$$
u R^{*} v \Longleftrightarrow(\mathrm{f} u) R^{*}(\mathrm{f} v)
$$

where $\mathrm{f} u=\left\{\mathrm{f}_{i} u_{i}\right\}_{i \in N}$. In what follows we use the notation $\mathrm{F}_{N}$, when we need to specify to which particular society $N$ the transforms of a class $F$ apply; when no ambiguity appears, the index $N$ will be omitted.

Under conditions imposed, the Arrovian social choice problem in the informational environment introduced by an invariance class $F$ can be equivalently described in terms of SWF $W$ which

1) is a continuous real-valued function $W: \mathbb{R}^{n} \rightarrow \mathbb{R}^{1}$, such that for any $c \in \mathbb{R}^{1}$, $W\left(c_{N}\right)=c$;

2) is nondecreasing ${ }^{2}$, i.e., for all $u, v \in \mathbb{R}^{n}$,

$$
u \geq v \Longrightarrow W(u) \geq W(v)
$$

3) is invariant under invariance transforms of class $\mathrm{F}$, i.e., for any $f \in \mathrm{F}$ and for all $u, v \in \mathbb{R}^{n}$,

$$
W(u) \geq W(v) \Longrightarrow W(\mathrm{f} u) \geq W(\mathrm{f} v) .
$$

For an invariance class $F$ to be a scale in the sense of the standard theory of measurement it has to satisfy the stronger condition of being a group (see Phanzagl [11]). Different scale types for individual utility measurement have been examined in the literature (Roberts [13], d'Aspremont [3], Bossert and Weymark [2]). Next we list the scales to be considered.

Ordinal Measurability (OM). $f \in F$ iff $f$ is a list of independent strictly increasing transforms $\mathrm{f}_{i}, i \in N$.

${ }^{2}$ This holds because $W$ is continuous and strictly increasing. 
Ordinal Measurability and Full Comparability (OFC). $f \in F$ iff $f$ is a list of identical strictly increasing transforms, i.e., for any real $t$ and all $i \in N, \mathrm{f}_{i}(t)=\mathrm{f}_{0}(t)$ where $f_{0}$ is a strictly increasing function independent of $i$.

Cardinal Measurability (CM). $f \in F$ iff $f$ is a list of independent strictly positive affine transforms, i.e., for any real $t$ and all $i \in N, \mathrm{f}_{i}(t)=\mathrm{a}_{i}+\mathrm{b}_{i} t$ for some real $\mathrm{a}_{i}$ and real $b_{i}>0$.

Cardinal Measurability and Unit Comparability (CUC). $f \in F$ iff $f$ is a list of strictly positive affine transforms with common unit, i.e., for any real $t$ and all $i \in N$, $\mathrm{f}_{i}(t)=\mathrm{a}_{i}+\mathrm{b} t$ for some real $\mathrm{a} i$ and $\mathrm{b}>0$ with $\mathrm{b}$ independent of $i$.

Cardinal Measurability and Full Comparability (CFC). $f \in F$ iff $f$ is a list of identical strictly positive affine transforms, i.e., for any real $t$ and all $i \in N \mathrm{f}_{i}(t)=$ $\mathrm{a}+\mathrm{b} t$ for some real $\mathrm{a}$ and $\mathrm{b}>0$, both independent of $i$.

The main concern of this paper is the situation when the entire society $N$ is partitioned into $m$ disjoint subgroups of individuals, i.e., $N=N_{1} \cup N_{2} \cup \cdots \cup N_{m}$ with $N_{i} \cap N_{j}=\emptyset$ for $i \neq j$. It is assumed that a SWF $W$ defined on $\mathbb{R}^{n}$ for different subgroups of variables indexed by $N_{k}, k \in\{1, \ldots, m\}$, may admit invariance transforms of different invariance classes $\mathrm{F} N_{k}$, which amounts to $W$ being invariant under transforms of a class $\mathrm{F}_{N}$ such that $\mathrm{F}_{N}=\left\{\mathrm{F}_{N_{k}}\right\}_{k=1}^{m}$, i.e., for every $\mathrm{f} \in \mathrm{F}_{N}$ for all $k \in\{1, \ldots, m\}, \mathrm{f}_{N_{k}}=\left\{\mathrm{f}_{i}\right\}_{i \in N_{k}} \in \mathrm{F}_{N_{k}}$. In other words $\mathrm{F}_{N}$ is the Cartesian product of the subgroup classes of transforms $\mathrm{F} N_{k}$. Notice that the class $\mathrm{F}_{N}$ meets the condition (1). But, in general, even if all invariant classes $\mathrm{F}_{N_{k}}$ are scales, $\mathrm{F}_{N}$ is not necessarily a scale: the condition of being a group may no longer hold. For example, a combination of CFC scales with a common zero is not a scale. In what follows we concentrate on mutually independent subgroup scales. The subgroup scales $\mathrm{F} N_{k}$, $k=1, \ldots, m$, are mutually independent, if for any distinct $k_{1}, k_{2} \in\{1, \ldots, m\}$, for all $i \in N_{k_{1}}$ and $j \in N_{k_{2}}$, there exist $\mathrm{f}_{i} \in \mathrm{F}_{N_{k_{1}}}$ and $\mathrm{f}_{j} \in \mathrm{F}_{N_{k_{2}}}$ such that $\mathrm{f}_{i}(t)=\mathrm{a}_{i}+\mathrm{b}_{i} t$ with $b_{i}>0$ and $f_{j}(t)=a_{j}+b_{j} t$ with $b_{j}>0$, where $a_{i} \neq a_{j}$ and $b_{i} \neq b_{j}$. Note that since $\mathrm{OM}$ and $\mathrm{CM}$ include the positive affine transforms, these classes are covered by the above definition as well. Mutual Independence preserves the group property and guarantees $\mathrm{F}_{N}$ to be the direct product of groups $\mathrm{F}_{N_{k}}$ when each of the $\mathrm{F} N_{k}$ is a group, i.e., it guarantees $\mathrm{F}_{N}$ to be a scale, if all $\mathrm{F}_{N_{k}}$ are scales. It should also be stressed that Mutual Independence is a property of the set of subgroup classes of transforms $\left\{\mathrm{F}_{N_{1}}, \ldots, \mathrm{F}_{N_{m}}\right\}$, not of individual transforms within these classes.

Introduce now some extra notation. By $n_{k}$ we denote the cardinality of $N_{k}$. It is obvious that ${ }_{k=1}^{m} n_{k}=n$. Let for any $u \in \mathbb{R}^{n}$ and all $k \in\{1, \ldots, m\}, u_{N_{k}}$ be a subvector of $u$ that belongs to $\mathbb{R}^{n_{k}}$ and is composed of components $u_{i}, i \in N_{k}$. $\mathbb{R}^{N_{k}}$ is a coordinate subspace of $\mathbb{R}^{n}$ induced by coordinates with indices from $N_{k}$, i.e.

$$
\mathbb{R}^{N_{k}}=\left\{v \in \mathbb{R}^{n} \mid v_{i}=0, i \notin N_{k}\right\} .
$$

For any $u \in \mathbb{R}^{n}$ and $k \in\{1, \ldots, m\}$, let

$$
\mathbb{R}^{N_{k}}(u)=\left\{u^{\prime} \in \mathbb{R}^{n} \mid u_{N \backslash N_{k}}^{\prime}=u_{N \backslash N_{k}}\right\}
$$


be a hyperplane of dimension $n_{k}$ parallel to coordinate subspace $\mathbb{R}^{N_{k}}$ and containing $u$. Obviously, $\mathbb{R}^{N_{k}}=\mathbb{R}^{N_{k}}(\mathbf{0})$ and $\mathbb{R}^{N_{k}}(u)=u+\mathbb{R}^{N_{k}}$.

Denote by

$$
D_{N_{k}}=\left\{u \in \mathbb{R}^{N_{k}} \mid u_{i}=u_{j}, i, j \in N_{k}, \& u_{i}=0, i \notin N_{k}\right\}
$$

the diagonal of a coordinate subspace $\mathbb{R}^{N_{k}}$, and let $L^{D}$ be a subspace of $\mathbb{R}^{n}$ spanned by the diagonals $D_{N_{k}}, k \in\{1, \ldots, m\}$. It is easy to see that every $u \in L^{D}, u=\left\{u_{i}\right\}_{i \in N}$, has the form $u_{i}=v_{k(i)}$, for some $v=v(u) \in \mathbb{R}^{m}$ and $k(i)$ defined by the relation $i \in N_{k(i)}$, i.e., all variables in $L^{D}$ indexed by the same subgroup of indices have the same value.

For any vector $u \in \mathbb{R}^{n}$ and any real $c$, denote by $u \| c_{N_{k}}$ the vector in $\mathbb{R}^{n}$ with components

$$
\left(u \| c_{N_{k}}\right)_{i}= \begin{cases}u_{i}, & i \in N \backslash N_{k}, \\ c, & i \in N_{k} .\end{cases}
$$

It is easy to see that $u \| c_{N_{k}}$ is an orthogonal projection of $u$ on the hyperplane $\mathbb{R}^{N \backslash N_{k}}\left(c_{N_{k}}\right)$. For any real $c$, let $\left(c_{N_{k}}, \mathbf{0}_{N \backslash N_{k}}\right)$ denote the vector in $\mathbb{R}^{n}$ with components

$$
\left(c_{N_{k}}, \mathbf{0}_{N \backslash N_{k}}\right)_{i}= \begin{cases}c, & i \in N_{k}, \\ 0, & i \in N \backslash N_{k} .\end{cases}
$$

We denote an orthogonal projection of the level surface $g(c)$ to the hyperplane $\mathbb{R}^{N_{k}}\left(c_{N}\right)$ by $g_{N_{k}}(c)$. For any two points $u, u^{\prime} \in \mathbb{R}^{n}, u \neq u^{\prime}$, let $l\left(u, u^{\prime}\right)$ and $r\left[u, u^{\prime}\right)$ be respectively a straight line passing through both points, $u$ and $u^{\prime}$, and a ray starting from $u$ and passing through $u^{\prime}$; moreover, by $r\left(u, u^{\prime}\right)=r\left[u, u^{\prime}\right) \backslash\{u\}$ we denote an open ray without its origin.

As usual, $\mathbb{R}_{+}^{n}=\left\{u \in \mathbb{R}^{n} \mid u_{i} \geq 0, i \in N, \& u \neq \mathbf{0}\right\}$ is the nonnegative orthant in $\mathbb{R}^{n}$. For the mean value of a vector $u \in \mathbb{R}^{n}$ we use the standard notation $\bar{u}$, i.e. $\bar{u}=\left(\begin{array}{c}n \\ i=1\end{array} u_{i}\right) / n$. Following [2], for any vector $u \in \mathbb{R}^{n}$, the fan generated by $u$ is

$$
Y(u)=\left\{u \in \mathbb{R}^{n}\left|u=q 1_{n}+\right| u, q \in \mathbb{R}, \mid \in \mathbb{R}_{+}\right\} .
$$

A subset $Y$ of $\mathbb{R}^{n}$ is a fan, if it is a fan generated by some $u \in \mathbb{R}^{n}$.

\section{Existence of a dictatorial subgroup}

Clearly, every continuous nondecreasing $n$-dimensional function that is determined only by variables with indices from one of the subgroups and that is invariant under invariance transforms proper to this subgroup of variables is a SWF. Below we study the situations for which such a form of a SWF is the only possible one, or equivalently, for which a dictatorial subgroup, i.e., a decisive coalition equal to one of the subgroups of individuals, must exist. The social ordering is then determined in accordance with the scale type of this dictatorial subgroup. 
Theorem 1. Let $N=N_{1} \cup N_{2} \cup \cdots \cup N_{m}, N_{i} \cap N_{j}=\emptyset$ for all $i \neq j$, and let a continuous nondecreasing function $W: \mathbb{R}^{n} \rightarrow \mathbb{R}^{1}$ with respect to variables indexed by $N_{k}$ be invariant under one of scales OM, OFM, CM, CUC, or CFC. Moreover, the subgroup scales are assumed to be mutually independent. Then there exists a unique integer $k \in\{1, \ldots, m\}$, such that for all $u \in \mathbb{R}^{n}, W$ has the form

$$
W(u)=W\left(u_{N_{k}}\right),
$$

i.e., $W$ is determined only by variables indexed by $N_{k}$, and besides is fully characterized by the scale type proper to this subset of variables.

Remark 2 Notice that any CFC transform at the same time is a transform of any of the OM, OFM, CM and CUC invariant classes. Hence, it is possible to simplify the statement of Theorem 1 by requiring only that the function $W(u)$ with respect to variables indexed by $N_{k}, k \in\{1, \ldots, m\}$, be invariant under mutually independent CFC transforms.

Theorem 1 allows us to construct a SWF characterization for various combinations of OM, OFM, CM, CUC and CFC independent subgroup utility scales on the basis of well-known results for social choice problems with the same measurement scales of individual utilities for the entire society.

In terms of level surfaces, the statement of Theorem 1 means that for any function $W(u)$, there exists a unique $k \in\{1, \ldots, m\}$ such that every level surface $g(c)$ is parallel to the coordinate subspace $\mathbb{R}^{N \backslash N_{k}}$. The latter is tantamount to $\mathbb{R}^{N \backslash N_{k}}(u) \subset g(W(u))$, for all $u \in \mathbb{R}^{n}$. It is not difficult to see that for the proof of the last inclusion, it is sufficient to show that every meet of set $g(W(u)) \cap \mathbb{R}^{N_{k}}(u)$, $k \in\{1, \ldots, m\}$, except one is a hyperplane of dimension $n_{k}$. For different combinations of mutually independent OM, CM and CUC scales, the result may be easily obtained based on the admissibility of the transform $f=\left\{f_{i}\right\}_{i \in N}$ :

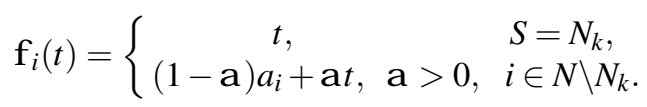

Indeed, for all combinations of OM, CM and CUC scales, for all $k \in\{1, \ldots, m\}$, every meet of set $g(W(u)) \cap \mathbb{R}^{N_{k}}(u)$ together with any two points contains the whole straight line passing through these points, and therefore has to be a hyperplane. So, for this case the proof of Theorem 1 is rather simple. However, if we append OFC and CFC scales, then the defined above transform $f$ is inadmissible for all combinations of scales, and not every meet of set $g(W(u)) \cap \mathbb{R}^{N_{k}}(u)$ is a hyperplane.

To prove Theorem 1, first, we show that every level surface $g(c)$ contains its own orthogonal projection $g_{N_{k}}(c)$ on the hyperplane $\mathbb{R}^{N_{k}}\left(c_{N}\right), k \in\{1, \ldots, m\}$, which in turn coincides with the meet of set $g(c) \cap \mathbb{R}^{N_{k}}\left(c_{N}\right)$ (Lemma 1). Next, in terms of these projections we derive a necessary and sufficient condition for a function $W(u)$ to be fully determined only by variables indexed by some fixed subgroup $N_{k}$ (Lemma 2). And finally, we prove that this condition holds under the hypothesis of the theorem (Lemma 3 and Lemma 4). 
Lemma 1. Any level surface $g(c)$ for all $k \in\{1, \ldots, m\}$ contains its own orthogonal projection on the hyperplane $\mathbb{R}^{N_{k}}\left(c_{N}\right)$, i.e.,

$$
g_{N_{k}}(c) \subset g(c),
$$

moreover, either $\operatorname{dim} g_{N_{k}}(c)=n_{k}$ or $\operatorname{dim}_{N_{k}}(c)=n_{k}-1$ and

$$
g_{N_{k}}(c)=g(c) \cap \mathbb{R}^{N_{k}}\left(c_{N}\right) .
$$

Proof. Fix some $k \in\{1, \ldots, m\}$. To prove (3) it will suffice to show that for every $u \in g(c), \quad u \| c_{N \backslash N_{k}} \in g(c)$. Let $u \in g(c)$. If $u \in \mathbb{R}^{N_{k}}\left(c_{N}\right)$, then $u \| c_{N \backslash N_{k}}=u$ and obviously, $u \| c_{N \backslash N_{k}} \in \mathrm{g}(c)$. Assume $u \notin \mathbb{R}^{N_{k}}\left(c_{N}\right)$. Due to Remark 1, $c_{N} \in \mathrm{g}(c)$. Take an admissible transform $f=\left\{f_{i}\right\}_{i \in N}$ :

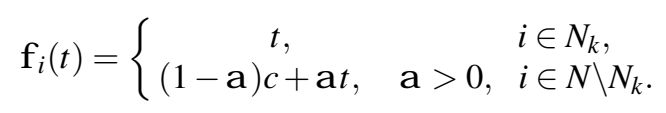

By (2) for all a $>0, W(\mathrm{f} u)=W\left(\mathrm{f} c_{N}\right)$. But for any a $>0, \mathrm{f} c_{N}=c_{N} \in \mathrm{g}(c)$. Hence, for all a $>0$, f $u \in \mathrm{g}(c)$, and moreover, since $u \notin \mathbb{R}^{N_{k}}\left(c_{N}\right), \mathrm{f} u$ corresponding to different a are different. If $\mathrm{a}=1, f u=u$, whence $r\left(u \| c_{N \backslash N_{k}}, u\right) \subset g(c)$. Therefore, every neighborhood of $u \| c_{N \backslash N_{k}}$ has a nonempty meet with $g(c)$. Whence by continuity of $W, u \| c_{N \backslash N_{k}} \in g(c)$. Since $W(u)$ is defined for every $u \in \mathbb{R}^{n}, W\left(u \| c_{N \backslash N_{k}}\right)$ is well defined. Assume $W\left(u \| c_{N \backslash N_{k}}\right)=a \neq c$. Because of continuity of $W$, there exists a neighborhood $S$ of $u \| c_{N \backslash N_{k}}$ such that $\left|W\left(u^{\prime}\right)-a\right|<|c-a| / 2$, for all $u^{\prime} \in S$, wherefrom $\left|W\left(u^{\prime}\right)-c\right|>|c-a| / 2$, for every $u^{\prime} \in S$. Hence, $W\left(u^{\prime}\right) \neq c$, for all $u^{\prime} \in S$. The obtained contradiction proves (3).

From the definition of orthogonal projection it follows directly that

$$
g_{N_{k}}(c) \subset \mathbb{R}^{N_{k}}\left(c_{N}\right)
$$

and

$$
g(c) \subset g_{N_{k}}(c)+\mathbb{R}^{N \backslash N_{k}} .
$$

Whence,

$$
\operatorname{dim} g_{N_{k}}(c) \leq n_{k}
$$

and

$$
\operatorname{dim} g(c) \leq \operatorname{dim} g_{N_{k}}(c)+\left(n-n_{k}\right) .
$$

Combining the last inequalities together with the equality $\operatorname{dimg}(c)=n-1$, we obtain

$$
n_{k}-1 \leq \operatorname{dim}_{N_{k}}(c) \leq n_{k}
$$

From the definition of orthogonal projection it also follows that

$$
g(c) \cap \mathbb{R}^{N_{k}}\left(c_{N}\right) \subset g_{N_{k}}(c) .
$$

From which together with (3) and (5), (4) follows immediately. 
Remark 3 Lemma 1 remains true under a coarser partition of $N$ into disjoint subgroups when a few subgroups $N_{k}, k \in\{1, \ldots, m\}$, may merge into one. It is worth noting that this remark concerns all subsequent propositions as well.

Remark 4 Due to the admissibility of the transform $\left\{f_{i}(t)=a+t\right\}_{i \in N}$ for all real a , the level surfaces $g(c)$ relevant to different $c$ can be obtained from each other by parallel shifts along the diagonal $D_{n}$. (This property was mentioned earlier in [13]). Wherefrom together with (4) it follows that for all real $c$ and $c^{\prime}$,

$$
g_{N_{k}}\left(c^{\prime}\right)=g_{N_{k}}(c)+\left(c^{\prime}-c\right)_{N},
$$

i.e., all projections $g_{N_{k}}(c)$ relevant to the same $k$ and different $c$ can be obtained from each other by parallel shifts along $D_{n}$.

Remark 5 Observe that $g_{N_{k}}(c)$ is a cone in $\mathbb{R}^{N_{k}}\left(c_{N}\right)$ with a top in $c_{N}$. Indeed, if $u^{\prime} \in g_{N_{k}}(c)$ and $u^{\prime} \neq c_{N}$, then there exists $u \in g(c), u \neq c_{N}$, such that $u^{\prime}=u \| c_{N \backslash N_{k}}$. Since $c_{N} \in g(c)$ and because of the admissibility of the transform $\left\{y_{i}(t)=(1-\right.$ a ) $c+a t\}_{i \in N}$ for all a $>0, r\left[c_{N}, u\right) \subset g(c)$. But the ray $r\left[c_{N}, u^{\prime}\right)$ is a projection of the ray $r\left[c_{N}, u\right)$ onto the hyperplane $\mathbb{R}^{N_{k}}\left(c_{N}\right)$. Thus, for every $u^{\prime} \in g_{N_{k}}(c)$ such that $u^{\prime} \neq c_{N}, r\left[c_{N}, u^{\prime}\right) \subset g_{N_{k}}(c)$, which proves that $g_{N_{k}}(c)$ is a cone. In particular, a cone $g_{N_{k}}(c)$ with $\operatorname{dim} g_{N_{k}}(c)=n_{k}$ may coincide with $\mathbb{R}^{N_{k}}\left(c_{N}\right)$. If $\operatorname{dim} g_{N_{k}}(c)=n_{k}-1$, it may be a hyperplane in $\mathbb{R}^{N_{k}}\left(c_{N}\right)$ passing through $c_{N}$.

Denote by $H_{N_{k}}(c)$ the cylinder $g_{N_{k}}(c)+\mathbb{R}^{N \backslash N_{k}}$.

Remark 6 As it was already noted in the proof of Lemma 1, for any real $c$ and all $k \in\{1, \ldots, m\}$,

$$
g(c) \subset H_{N_{k}}(c) .
$$

Lemma 2. A function $W$ for any $u \in \mathbb{R}^{n}$ has the form

$$
W(u)=W\left(u_{N_{k}}\right), \quad \text { for some } \quad k \in\{1, \ldots, m\},
$$

i.e. depends only on the variables $u_{i}$ with indices $i \in N_{k}$, if and only if there exists real c such that $\operatorname{dim} g_{N_{k}}(c)=n_{k}-1$.

Proof. I. Necessity. Clearly, for every real $c$

$$
g(c) \cap \mathbb{R}^{N_{k}}\left(c_{N}\right)=\left\{u \in \mathbb{R}^{N_{k}}\left(c_{N}\right) \mid W(u)=c\right\} .
$$

By hypothesis, for all $u \in \mathbb{R}^{n}$ and, in particular, for all $u \in \mathbb{R}^{N_{k}}\left(c_{N}\right), W(u)=$ $W\left(u_{N_{k}}\right)$. But for $u \in \mathbb{R}^{N_{k}}\left(c_{N}\right)$, the variables $u_{N_{k}}$ are intrinsic coordinates in $\mathbb{R}^{N_{k}}\left(c_{N}\right)$. Therefore and because of (4), the projection $g_{N_{k}}(c)$, being a subset of the $n_{k}$ dimensional hyperplane $\mathbb{R}^{N_{k}}\left(c_{N}\right)$, is characterized by the unique equality $W\left(u_{N_{k}}\right)=$ $c$ in the intrinsic coordinates of $\mathbb{R}^{N_{k}}\left(c_{N}\right)$, whence it follows that for every $c$, $\operatorname{dim} g_{N_{k}}(c)=n_{k}-1$.

II. Sufficiency. From (6) for all real $c$ and $c^{\prime}$,

$$
H_{N_{k}}\left(c^{\prime}\right) \cap \mathbb{R}^{N_{k}}\left(c_{N}\right)=g_{N_{k}}(c)+\left(\left(c^{\prime}-c\right)_{N_{k}}, \mathbf{o}_{N \backslash N_{k}}\right),
$$


i.e., for all $c^{\prime} \neq c$, every meet of set $H_{N_{k}}\left(c^{\prime}\right) \cap \mathbb{R}^{N_{k}}\left(c_{N}\right)$ is obtained from $g_{N_{k}}(c)$ by a parallel shift along the diagonal $D_{N_{k}}(c)$ of the hyperplane $\mathbb{R}^{N_{k}}\left(c_{N}\right)$,

$$
D_{N_{k}}(c)=\left\{u \in \mathbb{R}^{n} \mid u_{i}=u_{j}, i, j \in N_{k} \& u_{i}=c, i \notin N_{k}\right\} .
$$

If we show that for every $k \in\{1, \ldots, m\}$ such that $\operatorname{dim}_{N_{k}}(c)=n_{k}-1$, all parallel shifts of $g_{N_{k}}(c)$ along $D_{N_{k}}(c)$ in $\mathbb{R}^{N_{k}}\left(c_{N}\right)$ do not meet each other and cover the whole $\mathbb{R}^{N_{k}}\left(c_{N}\right)$, it will follow that cylinders $H_{N_{k}}(c)$ relevant to different $c$ do not meet and cover $\mathbb{R}^{n}$. On the other hand, since $W$ is defined on the entire $\mathbb{R}^{n}$, for every $u \in$ $\mathbb{R}^{n}$, there exists a level surface of $W$ containing $u$. Hence, because of (7) for every real $c, g(c)=H_{N_{k}}(c)$, which is the same as for all $u \in \mathbb{R}^{n}, W(u)=W\left(u_{N_{k}}\right)$. Thus, to complete the proof of sufficiency, it is enough to show that for every $k \in\{1, \ldots, m\}$ for which $\operatorname{dim} g_{N_{k}}(c)=n_{k}-1$, the parallel shifts of $g_{N_{k}}(c)$ along $D_{N_{k}}(c)$ in $\mathbb{R}^{N_{k}}\left(c_{N}\right)$ do not meet each other and cover $\mathbb{R}^{N_{k}}\left(c_{N}\right)$.

First, we show that for every $k \in\{1, \ldots, m\}$, the parallel shifts of $g_{N_{k}}(c)$ along $D_{N_{k}}(c)$ in $\mathbb{R}^{N_{k}}\left(c_{N}\right)$ cover $\mathbb{R}^{N_{k}}\left(c_{N}\right)$. For any $u \in \mathbb{R}^{n}$ the level surface $g(W(u))$ passes through $u$. Whence and because of Remark 1, every $g(W(u))$ is a cone with a top in $\{W(u)\}_{N} \in D_{n}$ and all level surfaces may be obtained from each other by parallel shifts along $D_{n}$. Therefore, through every point in any two-dimensional half-plane with a boundary $D_{n}$, denoted in the sequel by $\mathbb{R}_{ \pm}^{2}\left(D_{n}\right)$, passes a ray that starts in some $c_{N} \in D_{n}$ and belongs completely to $g(c)$. Moreover, since different level surfaces do not meet and are obtained from each other by parallel shifts along $D_{n}$, from every point $c_{N} \in D_{n}$, in any half-plane $\mathbb{R}_{ \pm}^{2}\left(D_{n}\right)$, there emanates a unique ray that belongs to $g(c)$ and that does not meet other level surfaces $g\left(c^{\prime}\right), c^{\prime} \neq c$. Parallel rays starting from different $c_{N} \in D_{n}$ and belonging to some half-plane $\mathbb{R}_{ \pm}^{2}\left(D_{n}\right)$ cover the entire $\mathbb{R}_{ \pm}^{2}\left(D_{n}\right)$. Hence, for every $c_{N} \in D_{n}$, in any two-dimensional plane $\mathbb{R}^{2}\left(D_{n}\right)$ passing through $D_{n}$ there are exactly two rays starting from $c_{N}$ and located in distinct half-planes of $\mathbb{R}^{2}\left(D_{n}\right)$ separated by $D_{n}$, i.e., in $\mathbb{R}_{+}^{2}\left(D_{n}\right)$ and $\mathbb{R}_{-}^{2}\left(D_{n}\right)$ respectively; in particular, these two rays may form a straight line meeting $D_{n}$ in $c_{N}$. A collection of mutually non-overlapping pairs of rays relevant to different level surfaces $g(c)$ covers $\mathbb{R}^{2}\left(D_{n}\right)$. Since every $u \in \mathbb{R}^{N_{k}}\left(c_{N}\right) \backslash D_{N_{k}}(c)$ and a straight line $D_{N_{k}}(c)$ determine unambiguously a twodimensional plane, a set of all two-dimensional planes $\mathbb{R}^{2}\left(D_{N_{k}}(c)\right) \subset \mathbb{R}^{N_{k}}\left(c_{N}\right)$ containing the diagonal $D_{N_{k}}(c)$ of $\mathbb{R}^{N_{k}}\left(c_{N}\right)$ covers $\mathbb{R}^{N_{k}}\left(c_{N}\right)$. Any plane $\mathbb{R}^{2}\left(D_{N_{k}}(c)\right)$ may be considered as a projection of a cylinder $\mathbb{R}^{2}\left(D_{N_{k}}(c)\right)+\mathbb{R}^{N \backslash N_{k}}$ on $\mathbb{R}^{N_{k}}\left(c_{N}\right)$. Since $D_{n} \subset \mathbb{R}^{2}\left(D_{N_{k}}(c)\right)+\mathbb{R}^{N \backslash N_{k}}$, every cylinder $\mathbb{R}^{2}\left(D_{N_{k}}(c)\right)+\mathbb{R}^{N \backslash N_{k}}$ is covered by a set of all two-dimensional planes $\mathbb{R}^{2}\left(D_{n}\right) \subset \mathbb{R}^{2}\left(D_{N_{k}}(c)\right)+\mathbb{R}^{N \backslash N_{k}}$. Observe that $D_{n} \| c_{N \backslash N_{k}}=D_{N_{k}}(c)$. Therefore, for each plane $\mathbb{R}^{2}\left(D_{n}\right) \subset \mathbb{R}^{2}\left(D_{N_{k}}(c)\right)+\mathbb{R}^{N \backslash N_{k}}$ that is not orthogonal to $\mathbb{R}^{2}\left(D_{N_{k}}(c)\right)$, a projection of $g(c) \cap \mathbb{R}^{2}\left(D_{n}\right)$ on $\mathbb{R}^{N_{k}}\left(c_{N}\right)$ consists of exactly two rays $\tilde{r}_{1}, \tilde{r}_{2} \subset g_{N_{k}}(c)$ starting from $c_{N} \in D_{N_{k}}(c)$ and belonging to the different half-planes $\mathbb{R}_{+}^{2}\left(D_{N_{k}}(c)\right)$ and $\mathbb{R}_{-}^{2}\left(D_{N_{k}}(c)\right)$ of the plane $\mathbb{R}^{2}\left(D_{N_{k}}(c)\right)$ that are separated by $D_{N_{k}}(c)$, i.e., $\tilde{r}_{1} \subset \mathbb{R}_{+}^{2}\left(D_{N_{k}}(c)\right), \tilde{r}_{2} \subset \mathbb{R}_{-}^{2}\left(D_{N_{k}}(c)\right)$. Any plane orthogonal to $\mathbb{R}^{2}\left(D_{N_{k}}(c)\right)$ maps completely on $D_{N_{k}}(c)$. Under parallel shifts along $D_{N_{k}}(c)$, rays $\tilde{r}_{1}$ and $\tilde{r}_{2}$ cover the entire plane $\mathbb{R}^{2}\left(D_{N_{k}}(c)\right)$, while the collection of all shifts of $g_{N_{k}}(c)$ along $D_{N_{k}}(c)$ covers the hyperplane $\mathbb{R}^{N_{k}}\left(c_{N}\right)$. 
To show that for every $k \in\{1, \ldots, m\}$ for which $\operatorname{dim}_{N_{k}}(c)=n_{k}-1$, parallel shifts of $g_{N_{k}}(c)$ along $D_{N_{k}}(c)$ in $\mathbb{R}^{N_{k}}\left(c_{N}\right)$ do not meet, it suffices to show that every half-plane $\mathbb{R}_{ \pm}^{2}\left(D_{N_{k}}(c)\right)$ contains a ray belonging to $g_{N_{k}}(c)$ and solely one. Assume the contrary, and let at least two rays $r_{1}, r_{2} \subset g_{N_{k}}(c) \cap \mathbb{R}_{ \pm}^{2}\left(D_{N_{k}}(c)\right)$. Then due to continuity of the level surface $g(c)$ and continuity of the projection mapping $\operatorname{Pr}: \mathbb{R}^{n} \rightarrow \mathbb{R}^{N_{k}}\left(c_{N}\right)$, the piece of a half-plane $\mathbb{R}_{ \pm}^{2}\left(D_{N_{k}}(c)\right)$ between rays $r_{1}$ and $r_{2}$ also belongs to $g_{N_{k}}(c)$ as well, which is impossible since by hypothesis, $\operatorname{dim} g_{N_{k}}(c)=n_{k}-1$.

Remark 7 The necessary and sufficient condition in Lemma 2 may be restated equivalently in terms of cylinders $H_{N_{k}}(c)$. Indeed, the equality

$$
\operatorname{dim} g_{N_{k}}(c)=n_{k}-1, \quad \text { for some } k \in\{1, \ldots, m\},
$$

is tantamount to the equality

$$
g(c)=H_{N_{k}}(c), \quad \text { for the same } k .
$$

Lemma 3. For every level surface $g(c)$, if for some $k \in\{1, \ldots, m\}$

1) $\operatorname{dim} g_{N_{k}}(c)=n_{k}-1$, then $g_{N \backslash N_{k}}(c)=\mathbb{R}^{N \backslash N_{k}}\left(c_{N}\right)$ and for all $k^{\prime} \in\{1, \ldots, m\}$, $k^{\prime} \neq k, g_{k_{k^{\prime}}}(c)=\mathbb{R}^{N_{k^{\prime}}}\left(c_{N}\right)$;

2) $\operatorname{dim} g_{N_{k}}(c)=n_{k}$, then $g_{N \backslash N_{k}}(c) \neq \mathbb{R}^{N \backslash N_{k}}\left(c_{N}\right)$,

and furthermore, if $g_{N_{k}}(c)=\mathbb{R}^{N_{k}}\left(c_{N}\right)$, then $\operatorname{dim} g_{N \backslash N_{k}}(c)=n-n_{k}-1$,

$$
\text { while if } \mathrm{g}_{N_{k}}(c) \neq \mathbb{R}^{N_{k}}\left(c_{N}\right) \text {, then } \operatorname{dim} g_{N \backslash N_{k}}(c)=n-n_{k} \text {. }
$$

Proof. To prove the first statement, assume that $\operatorname{dim}_{N_{k}}(c)=n_{k}-1$, for some $k \in$ $\{1, \ldots, m\}$. By (4) and (8), for all $k \in\{1, \ldots, m\}$

$$
g_{N \backslash N_{k}}(c)=g(c) \cap \mathbb{R}^{N \backslash N_{k}}\left(c_{N}\right)=H_{N_{k}}(c) \cap \mathbb{R}^{N \backslash N_{k}}\left(c_{N}\right)=\mathbb{R}^{N \backslash N_{k}}\left(c_{N}\right) .
$$

Similarly for all $k^{\prime} \in\{1, \ldots, m\}, k^{\prime} \neq k$,

$$
g_{N_{k^{\prime}}}(c)=g(c) \cap \mathbb{R}^{N_{k^{\prime}}}\left(c_{N}\right)=H_{N_{k}}(c) \cap \mathbb{R}^{N_{k^{\prime}}}\left(c_{N}\right)=\mathbb{R}^{N_{k^{\prime}}}\left(c_{N}\right) .
$$

Prove now the second one. Assume the contrary that $g_{N \backslash N_{k}}(c)=\mathbb{R}^{N \backslash N_{k}}\left(c_{N}\right)$. Then because of (3), $\mathbb{R}^{N \backslash N_{k}}\left(c_{N}\right) \subset g(c)$, which is equivalent to $W(u)=W\left(u_{N_{k}}\right)$. Whence, by Lemma $2, \operatorname{dim} g_{N_{k}}(c)=n_{k}-1$, which contradicts to the hypothesis.

Next, from Lemma 1 and Remark 3 it follows that either $\operatorname{dim} g_{N \backslash N_{k}}(c)=n-n_{k}$, or $\operatorname{dim} g_{N \backslash N_{k}}(c)=n-n_{k}-1$. If $\operatorname{dim}_{N \backslash N_{k}}(c)=n-n_{k}-1$, then by the Remark 7, $g(c)=H_{N \backslash N_{k}}(c)$. Obviously,

$$
H_{N \backslash N_{k}}(c) \cap \mathbb{R}^{N_{k}}\left(c_{N}\right)=\mathbb{R}^{N_{k}}\left(c_{N}\right),
$$

i.e.,

$$
g(c) \cap \mathbb{R}^{N_{k}}\left(c_{N}\right)=\mathbb{R}^{N_{k}}\left(c_{N}\right),
$$

whence by (4), $g_{N_{k}}(c)=\mathbb{R}^{N_{k}}\left(c_{N}\right)$. 
Further, if we suppose $g_{N_{k}}(c)=\mathbb{R}^{N_{k}}\left(c_{N}\right)$ and repeat the latter arguments, then because of (3) and Lemma 2, we arrive at $\operatorname{dim} g_{N \backslash N_{k}}(c)=n-n_{k}-1$.

Remark 8 Because of Remark 3, the validity of the second statement in the second point of Lemma 3 can be obtained directly from the first point as well.

From the first statement of Lemma 3 applying the induction argument with respect to the number $m$ of subgroups $N_{k}$ in the partition of $N$, we derive the next corollary.

Corollary 1. For any level surface $g(c)$ not every projection $g_{N_{k}}(c), k \in\{1, \ldots, m\}$, coincides with the corresponding hyperplane $\mathbb{R}^{N_{k}}\left(c_{N}\right)$.

Lemma 4. For every level surface $g(c)$, for any $k \in\{1, \ldots, m\}$, a projection $g_{N_{k}}(c)$ either coincides with a hyperplane $\mathbb{R}^{N_{k}}\left(c_{N}\right)$ or $\operatorname{dim} g_{N_{k}}(c)=n_{k}-1$.

Proof. From the first statement of Lemma 3, if $\operatorname{dim}_{N_{k}}(c)=n_{k}$ and $g_{N_{k}}(c) \neq$ $\mathbb{R}^{N_{k}}\left(c_{N}\right)$, then $\operatorname{dim}_{N \backslash N_{k}}(c)=n-n_{k}$. It follows that there exist a real $\mathrm{e}>0$ and two points $u_{1} \in g_{N_{k}}(c), u_{2} \in g_{N \backslash N_{k}}(c)$, such that $u_{1}^{\mathrm{e}}=u_{1}+\left(\mathrm{e}_{N_{k}}, \mathbf{0}_{N \backslash N_{k}}\right) \in \mathrm{g}_{N_{k}}(c)$ and $u_{2}^{\mathrm{e}}=u_{2}+\left(\mathbf{0}_{N_{k}}, \mathrm{e}_{N \backslash N_{k}}\right) \in \mathrm{g}_{N \backslash N_{k}}(c)$. Moreover, by Lemma 1 and Remark 3 , $u_{1}, u_{1}^{\mathrm{e}}, u_{2}, u_{2}^{\mathrm{e}} \in \mathrm{g}(c)$. Consider the admissible transform $\mathrm{f}=\left\{\mathrm{f}_{i}\right\}_{i \in N}$ :

$$
\mathrm{f}_{i}(t)=\left\{\begin{array}{cl}
t+\mathrm{e}, & i \in N_{k} \\
t, & i \in N \backslash N_{k}
\end{array}\right.
$$

By (2), $W\left(\mathrm{f} u_{1}\right)=W\left(\mathrm{f} u_{2}^{\mathrm{e}}\right)$. Then notice that $\mathrm{f} u_{1}=u_{1}^{\mathrm{e}} \in \mathrm{g}(c)$. Hence, $\mathrm{f} u_{2}^{\mathrm{e}} \in \mathrm{g}(c)$. But $f u_{2}^{\mathrm{e}}=u_{2}+\mathrm{e}_{N}$, whence since $u_{2} \in \mathrm{g}(c)$ and since all level surfaces $g\left(c^{\prime}\right)$ for different $c^{\prime}$ can be obtained from each other by parallel shifts along $D_{n}, \mathrm{f} u_{2}^{\mathrm{e}} \in \mathrm{g}(c+$ e ). But for $f u_{2}^{e} \in g(c)$, the latter is impossible.

Remark 9 From Remark 4 it follows that, if for some $c$ and $k \in\{1, \ldots, m\}$, the statement of Lemma 3 or of Lemma 4 holds true, then for the same $k$ it holds true for all $c^{\prime} \neq c$.

Proof of Theorem 1 From Corollary 1 and Lemma 4 it follows that for some $k \in$ $\{1, \ldots, m\}, \operatorname{dim}_{N_{k}}(c)=n_{k}-1$. Moreover, by the first statement of Lemma 3 , this $k$ is unique. Whence together with Lemma 2 we obtain the validity of Theorem 1.

Acknowledgements The paper was partially written during the author's 2008 research stay in Tilburg Center for Logic and Philosophy of Science (TiLPS, Tilburg University) whose hospitality and support are highly appreciated.

\section{References}

1. Arrow KJ (1951; 2nd ed 1963) Social choice and individual values. Wiley, New York

2. Bossert W, Weymark JA (2004) Utility in social choice. In Barberá S, Hammond PJ, Seidl C (eds) Handbook of utility theory, Vol. 2. Kluwer Academic Publishers: 1099-1178 
3. d'Aspremont C (1985) Axioms for social welfare orderings. In Hurwicz L, Schmeidler D, Sonnenschein $\mathrm{H}$ (eds) Social goals and social organizations: essays in memory of Elisha Pazner. Cambridge University Press, Cambridge: 19-76

4. d'Aspremont C and Gevers L (1977) Equity and the informational basis of collective choice: Review of Economic Studies 44: 199-209

5. Debreu G (1954) Representation of a preference ordering by a numerical function. In Thrall RM, Coombs CH, Davis RL (eds) Decision processes. Wiley, New York: 159-165

6. Hammond PJ (1979) Equity in two person situations: some consequences: Econometrica 47: $1127-1135$

7. Khmelnitskaya AB (1996) Social choice problems with different scales of individual welfares measurement for different subgroups of individuals. In Kleinschmidt P, Bachem A, Derigs U, Fischer D, Leopold-Wildburger U, Möring R (eds) Operations research proceedings 1995. Springer-Verlag, Berlin: 252-257

8. Khmelnitskaya AB (1999) Social welfare orderings for different subgroup utility scales. Discussion paper \#198, Center for Rationality and Interactive Decision Theory at The Hebrew University of Jerusalem.

9. Khmelnitskaya AB (2002) Social welfare functions for different subgroup utility scales. In Tangian A, J. Gruber J (eds) Lecture Notes in Economics and Mathematical Systems, vol. 510. Springer-Verlag, Berlin: 515-530

10. Khmelnitskaya AB, Weymark JA (2000) Social choice with independent subgroup utility scales: Social Choice and Welfare 17: 739-748

11. Phanzagl J (1971): Theory of measurement (2nd ed). Physica-Verlag, Würzburg-Wien.

12. Roberts KWS (1980) Possibility theorems with interpersonally comparable welfare levels: Rev Econ Studies 47: 409-420

13. Roberts KWS (1980) Interpersonal comparability and social choice theory: Rev Econ Studies 47: 421-439

14. Sen AK (1970) Collective choice and social welfare. Holden-Day, San Francisco

15. Tsui K-Y and Weymark JA (1997) Social welfare orderings for ratio-scale measurable utilities: Economic Theory 10: 241-256

16. Yanovskaya EB (1988) Social choice functions for different scales of individual preference measurement (in Russian): Annals of VNIISI (All-Union Research Institute for System Studies, Moscow) 6: 64-76

17. Yanovskaya EB (1989) Group choice rules in problems with comparisons of individual preferences: Automation and Remote Control 50: 822-830 (translated from the 1989 Russian original that appeared in Avtomatika i Telemekhanika 6: 129-138) 